\title{
INCIDÊNCIA DE DISFAGIA OROFARÍNGEA APÓS ACIDENTE VASCULAR ENCEFÁLICO EM HOSPITAL PÚBLICO DE REFERÊNCIA
}

\author{
Arthur Oscar Schelp¹, Paula Cristina Cola², Ana Rita Gatto², Roberta Gonçalves da Silva³ Lídia Raquel de \\ Carvalho
}

RESUMO - A doença vascular encefálica (AVE) é a principal causa de morte no Brasil. As seqüelas em indivíduos pós-acidente vascular encefálico incluem distúrbios motores, distúrbios de fala ou de linguagem e distúrbios de deglutição. A disfagia orofaríngea ocorre em cerca de $50 \%$ dos pacientes com AVE. Este estudo teve por objetivo determinar a incidência da disfagia apósAVE. Foram avaliados todos os pacientes que deram entrada em hospital universitário de referência no período de um ano, tão logo apresentassem condições para avaliação clínica, fonoaudiológica e neurológica (102 pacientes), com análise objetiva da deglutição (61 pacientes). Foi observada incidência de disfagia em 76,5\% dos pacientes avaliados clinicamente, este percentual elevando-se a $91 \%$ com avaliação videofluoroscópica. A alta incidência de disfagia observada neste estudo que avaliou pacientes com amplo espectro de gravidade, em diferentes fases de recuperação, ressalta a importância de equipe multidisciplinar, incluindo fonoaudiólogos capacitados, para avaliar os distúrbios da deglutição nos diversos momentos de recuperação dos AVEs.

PALAVRAS-CHAVE: epidemiologia, disfagia orofaríngea, acidente vascular encefálico.

\begin{abstract}
Incidence of oropharyngeal dysphagia associated with stroke in a regional hospital in São Paulo State - Brazil
ABSTRACT - Cerebrovascular disease is recognized as to be associated with the highest mortality rate in Brazil. Dysphagia, speech and language disturbances are common consequences of the high incidence of stroke. Dysphagia is known to occur in at least $50 \%$ of the patients with acute stroke. The study is designed to stablish the incidence of stroke in a reference hospital. One hundred and two consecutive patients admitted between J anuary 2001 and January 2002 underwent a neurological examination with dysphagia specific analysis, performed by speech/dysphagia professionals as soon as they have clinical conditions. The localization of the lesions are determined by computer tomography or magnetic resonance image of the brain. Sixty one patients underwent videofluorscopic evaluation of swallowing. There was detected oropharyngeal dysphagia in 78 patients $(76.5 \%)$ if the examination was limited to the clinical evaluation. Neverthless, if complemented by videofluorscopic evaluation, the incidence grows to $90 \%$. The explanation for the high incidence observed in this study could be pointed to the fact that dysphagia was registered on different times of the convalescence period. The data reinforces the importance of repeated evaluations made by different professionals of the sttaff involved in acute stroke attendance.
\end{abstract}

KEY WORDS: epidemiology, oropharyngeal dysphagia, stroke.

A disfagia é reconhecida como um dos principais fatores de risco para ocorrência de pneumonia aspirativa ${ }^{1,2}$, uma das complicações mais freqüentes dos acidentes vasculares do encéfalo (AVEs), principal causa de morte no Brasil ${ }^{3}$. Barreto ${ }^{4}$ chamava a atenção para a freqüência e gravidade de disfagia após AVEs e para a necessidade do pronto reconhecimento e tratamento adequado.

A prevalência dos distúrbios da deglutição tem sido avaliada em pacientes internados, incluindo quadros nosológicos indiscriminados, com percentual em torno de $12 \% \%^{5}$. Diversos estudos que abordaram os aspectos epidemiológicos e história natural da disfagia, associada a quadros vasculares encefálicos agudos, apontam para uma incidência em torno de $50 \%$ para distúrbios da deglutição ${ }^{2,6-8}$. Na maioria destes, os pacientes foram inquiridos para presença de disfagia já na entrada, com avaliação de deglutição clínica e videofluoroscópica, nos

Hospital das Clínicas da Universidade Estadual de São Paulo, Botucatu SP, Brasil (UNESP): PProfessor Doutor Departamento de Neurologia e Psiquiatria, UNESP; ${ }^{2}$ Fonoaudiólogas do Departamento de Neurologia e Psiquiatria - UNESP; 3 Professora Doutora do Departamento de Fonoaudiologia da UNESP (M arília SP, Brasil); ${ }^{4}$ Professora Doutora Departamento de Bioestatística UNESP. Projeto Fapesp: no 01/08962-9

Recebido 22 Agosto 2003, recebido na forma final 17 Dezembro 2003. Aceito 14 Janeiro 2004.

Dr. Arthur Oscar Schelp - Departamento de Neurologia e Psiquiatria FM/UNESP - Caixa Postal 540 - 18618-970 Botucatu SP - Brasil. 
primeiros dias após internação. Os protocolos excluíam os pacientes com as formas mais graves, afásicos ou com sonda naso-enteral. Por outro lado, análises de pacientes préselecionados, demonstraram disfagia em 65 a $81 \%$ dos enfermos com AVEs localizados em tronco encefálico $0^{9,10}$. Não encontramos referências a estudos epidemiológicos da disfagia em AVE no Brasil. Estudos, como o de Silva e cols. ${ }^{11}$, avaliaram os graus de disfagia correlacionados com topografia radiológica. A mortalidade da doença no Brasil justifica estudos epidemiológicos, que considerem as características demográficas e populacionais do país.

Este estudo teve por objetivo avaliar indivíduos pósacidente vascular encefálico, isquêmico ou hemorrágico, determinando a incidência de disfagia.

\section{MÉTODO}

No período entre 10 de janeiro de 2002 e 10 de janeiro de 2003, deram entrada no Hospital das Clínicas da Faculdade de Medicina de Botucatu, 241 pacientes, com diagnóstico de AVE, hemorrágico e isquêmico. Cento e trinta e nove pacientes foram excluídos da análise, em razão de transferência para outras unidades, óbito, coma persistente, sedação e intercorrências clínicas que impediram avaliação mesmo após quatro semanas. Entre estes também incluem-se aqueles com quadros isquêmicos e sintomas neurológicos transitórios, ou foram encaminhados para seguimento ambulatorial, em outras unidades de atendimento do Sistema Único de Saúde.

Cento e dois pacientes foram avaliados clinicamente, por neurologistas e por fonoaudiólogos. 0 tempo mediano referente a avaliação da deglutição foi 6 dias (faixa 0-31dias). Destes, 98 realizaram tomografia computadorizada (TC) e ou ressonância magnética (RM) e 61 a videofluoroscopia da deglutiç̧ão. 0 tempo mediano foi 12 dias (faixa 0 a 3 meses). 0 estudo incluiu avaliação da deglutição dos pacientes com diagnóstico de AVE, ingressos no hospital, no período de 12 meses. Foram incluídos no protocolo os pacientes com sintomas de AVE, iniciados havia não mais do que 72 horas. Foram excluídos os pacientes submetidos a cirurgia para drenagem de hematoma intracerebral e aqueles com AVE, com sintomas neurológicos transitórios. Os pacientes transferidos para outras unidades e que faleceram nos primeiros três dias, não foram incluídos no estudo. Não foram incluídos na análise os pacientes com distúrbios da consciência e comprometimento cognitivo persistente, que impossibilitaram avaliação clínica/videofluoroscópica, interferindo na percepção do paciente e nos procedimentos de avaliação, no período do estudo.

0 protocolo de estudo foi aprovado pelo Comitê de Ética em Pesquisa Médica - CREM ESP, Hospital das Clínicas, UNESP. Todos os pacientes incluídos no protocolo de estudo tiveram ciência e deram consentimento livre e esclarecido. Na impossibilidade destes, o termo foi assinado por familiar ou responsável.

A investigação incluiu avaliação, por fonoaudiólogos e neurologistas, na entrada e assim que 0 doente estivesse atendendo a ordens simples, com quadro clínico estável, permitindo avaliação da deglutição. Avaliação complementar incluiu exames de TC de crânio e/ou RM e videofluoroscopia da deglutição.

Avaliação clínico-fonoaudiológica constou da aplicação de protocolo englobando dinâmica da deglutição, procedimentos diagnósticos e terapêutica fonoaudiológica, além da avaliação objetiva da deglutição (videofluoroscopia da deglutição). Os critérios para a introdução de dieta foram estabelecidos a partir do desempenho da deglutição em cada indivíduo.

Dentre os pacientes avaliados $30(31,3 \%)$ eram do gênero feminino e $66(68,8 \%)$ do masculino, com média de idade de 62,2 anos (32 a 92 anos de idade).

\section{RESULTADOS}

Dentre os 102 pacientes avaliados clinicamente pela fonoaudiologia, $76,5 \%$ apresentaram disfagia no momento da avaliação (Fig 1). É possível observar que pelo menos um terço dos pacientes foram avaliados havia pelo menos seis dias da instalação do AVE. Dos pacientes avaliados clinicamente pela fonoaudiologia, 24 pacientes não apresentaram disfagia (Fig 2). Dentre os pacientes que tinham disfagia, a maioria apresentava a forma leve. Análise da distribuição das disfagias, segundo gravidade, com avaliação videofluoroscópica, realizada em 61 pacientes, resultou em distribuição semelhante ao grupo submetido a avaliação clínico-fonoaudiológica (Fig 3). Dentre estes, 12 pacientes que não apresentaram disfagia, tinham sinais de disfunção no exame videofluoroscópico da deglutição, elevando o percentual de disfagia para $91 \%$.

\section{DISCUSSÃO}

Gordon e col. ${ }^{12}$ realizaram estudo prospectivo para registrar a incidência de disfagia em 100 pacientes admitidos em período de 6 meses, em hospital geral. Determinaram a presença de disfagia em $45 \%$ dos pacientes. Se compararmos com o percentual de $76,5 \%$, encontrado no presente estudo, fica o questionamento para as razões para tanta discrepância. Naquele estudo, os autores definiram como presença de disfagia, a incapacidade para ingerir $50 \mathrm{~mL}$ de água, ou presença de tosse em duas solicitações consecutivas para ingestão de igual quantidade de líquido. Neste, a avaliação clínica da deglutição foi realizada com consistências e volumes

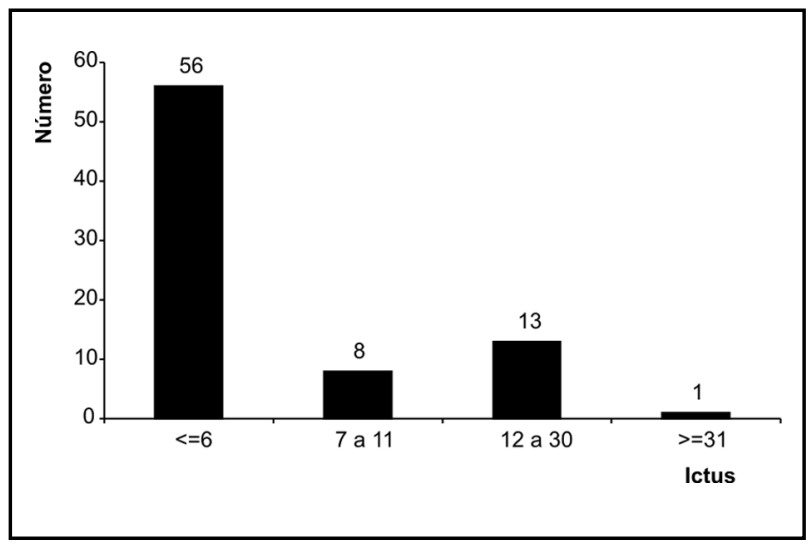

Fig 1. Distribuição da freqüência dos pacientes disfágicos relacionados ao ictus. Avaliação clínica fonoaudiológica. 


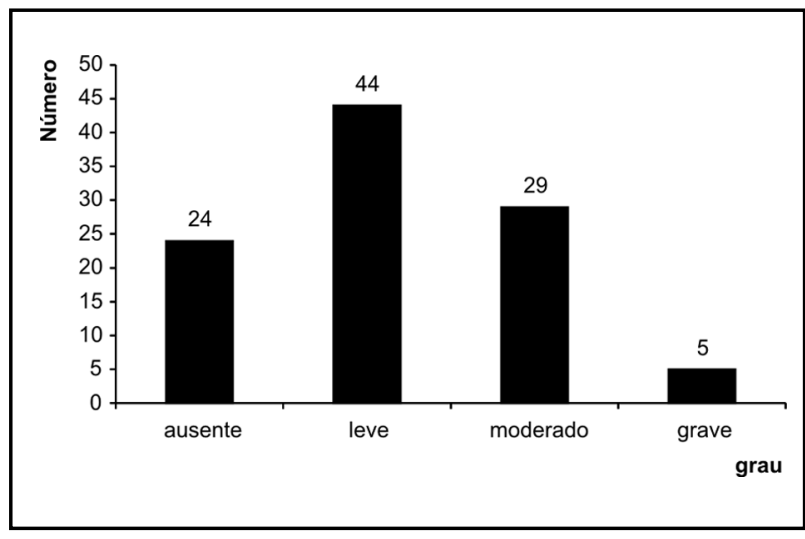

Fig 2. Distribuição dos pacientes segundo grau de disfagia - avaliação clínica fonoaudiológica.

(de 3 a $10 \mathrm{~mL}$ ) variados, incluindo pastosa fina, líquida e sólida. Foi avaliado comprometimento da fase oral (i.e. escape oral anterior) e da fase faríngea (i.e. alteração na ausculta cervical). A metodologia daquele estudo foi distinta da empregada nesta análise, tanto no que se refere à gravidade dos pacientes estudados, e que foram avaliados de 48 a 96 horas após o ictus, como nos critérios para determinação de disfagia. Já no estudo de Smithhard e col. ${ }^{7}$, que avaliaram 121 pacientes com diagnóstico de AVE, foram excluídos os pacientes admitidos mais de 24 horas após o ictus, doença grave concomitante (definida como maligna, i.e. risco de morte) ou cujos termos de consentimento não era passível de obtenção ou recusado. Estes autores registraram disfagia, no dia da admissão hospitalar, em 61 (51\%) pacientes. Ao não incluir pacientes graves, de avaliação impossível nas primeiras horas, os autores deixam de considerar os casos de disfagia perceptíveis quando o paciente recobra a consciência, sai do sistema de respiração controlada ou assistida e só então permite avaliação para presença ou ausência de disfagia.

Outros autores, como Mann e col. ${ }^{13} \mathrm{em}$ análise de 128 pacientes, no período de um ano, encontraram disfagia, determinada clinicamente, em 65 (51\%) pacientes. No estudo referido, foi realizada avaliação no leito, com mediana de 3 dias. Os pacientes do presente estudo foram avaliados de 1 a 60 dias após entrada no hospital, com mediana de 4 dias, 0 que demonstra, de forma inequívoca, que os pacientes analisados, apresentavam formas mais graves, tinham permanência hospitalar mais prolongada com avaliação tão logo apresentassem condições, com critérios de inclusão mais abrangentes.

Não foi possível comparar o percentual estabelecido, de $76,5 \%$, com outros, pela diferença nos critérios de seleção, mais abrangente, com avaliações em períodos mais prolongados em relação ao ictus, o que possibilitou avaliar pacientes com amplo espectro de formas clínicas, desde as mais leves até aquelas de maior gravidade.

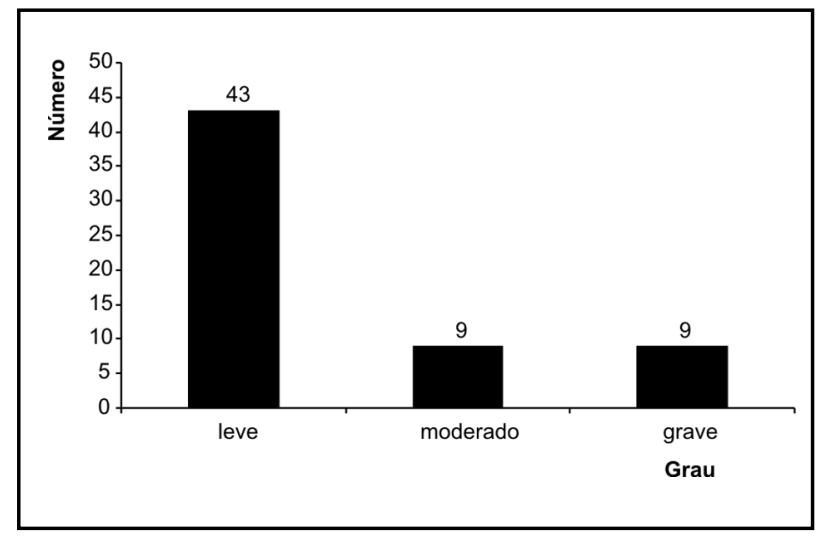

Fig 3. Distribuição dos pacientes segundo grau de disfagia - avaliação videofluoroscópica da deglutição.

0 presente estudo lança novas questões sobre a metodologia a ser empregada para obter os dados da epidemiologia de disfagia em pacientes com AVE agudo, que dão entrada em hospitais e que permanecem internados por períodos diferentes. $A$ incidência de disfagia foi bem maior que as registradas até então, dando uma dimensão clara da gravidade e importância do diagnóstico de disfagia, assim que os pacientes apresentassem condições clínicas, e não somente na fase aguda, ao dar entrada no hospital. Não é possível, no entanto, afirmar que a alta incidência observada neste estudo seja devida somente aos critérios de seleção mais abrangentes. A pequena disponibilidade de leitos, em relação a grande demanda, fez com que alguns pacientes portadores de infarto vascular encefálico, com sinais neurológicos transitórios, fossem avaliados ambulatorialmente, e que muitos pacientes conscientes, cooperantes e com melhor prognóstico, tivessem sua transferência para hospitais de menor porte.

A presença de disfagia, constatada na análise videofluoroscópica, em 61 pacientes, dos quais 12 não tinham diagnóstico clínico de disfagia, elevou a incidência a valores acima de $90 \%$. A maior sensibilidade do exame videofluoroscópico permite detectar tanto formas leves de disfagia, como alterações de fase faríngea dificilmente visualizadas pela avaliação clínica ${ }^{14}$. Mann e col. ${ }^{13}$ estudaram 161 pacientes pós AVE, clínica e videofluoroscopicamente, e também encontraram maior incidência de disfagia através do exame objetivo (64\%), em comparação com os $51 \%$ detectados na avaliação clínica.

Pelo exposto, é possível emitir algumas considerações relativas à epidemiologia da disfagia, em hospital de referência, com análise videofluoroscópica e neuroimagem:

Parece não restar dúvidas de que a alta incidência de disfagia, nas fases aguda e de recuperação dosAVEs, representa co-fator de mortalidade e morbidade, com todos os custos agregados para a sociedade, e que deve haver preocupação específica com a questão. 
0 diagnóstico de disfagia não deve ficar restrito às fases agudas dos AVES, pois, como foi demonstrado, há aumento considerável da incidência se as avaliações forem repetidas a medida em que o paciente apresente recuperação do quadro clínico, permitindo detecção de disfagia.

0 fonoaudiólogo deve fazer parte da equipe multidisciplinar que atua no diagnóstico e recuperação de pacientes com AVE, tanto na fase aguda como nas fases mais tardias.

Agradecimento - Ao colega de trabalho André Augusto Spadotto, responsável pelo processamento de dados do Departamento. de Neurologia e Psiquiatria, pelo apoio às nossas atividades de pesquisa.

\section{REFERÊNCIAS}

1. Mann G, Graeme J, Hankey GJ. Initial clinical and demographic predictors of swallowing impairment following acute stroke. Dysphagia 2001;16:208-215.

2. Smithard DG, $\mathrm{O}^{\prime}$ Neill PA, Park $\mathrm{Cl}$, et al. Complications and outcome after acute stroke. Stroke 1996;27:1200-1204.

3. Lessa I. Epidemiologia das doenças cerebrovasculares no Brasil. Rev Soc Cardiol Estado S. Paulo 1999;4:509-518.

4. Barreto PM. Cuidados especiais com as perturbações respiratórias e digestivas decorrentes do acidentes vasculares encefálicos. In Melaragno R Filho. (ed). Afecções vasculares cerebrais: diagnóstico e tratamento. São Paulo: Livraria Luso-Espanhola e Brasileira1, 959:157-180.

5. Groher ME, Bukatman R. The prevalence of swallowing disorders in two teaching hospitals. Dysphagia 1986;1:3-6.

6. Smithard DG, O'Neill PA, England RE, et al. The natural history of dysphagia following a stroke. Dysphagia 1997;12:188-194.

7. Ekberg NH, Hindfeldt OR. Dysphagia in stroke: a prospective study quantitative aspects of swallowing in dysphagic patients. Dysphagia 1998;13:32-38.

8. Gresham, SL. Clinical assessment and management of swallowing difficulties after stroke. Med J Aust 1990;153:397-399.

9. Memg NH, Wang TG, Lien IN. Dysphagia in patients with brainstem stroke: incidence and outcome. Am J Phys Med Rehabil 2000;79:170-175.

10. Horner J, Buoyer FG, Alberts MJ, Helms MJ. Dysphagia following brainstem stroke: clinical correlates and outcome. Arch Neurol 1991;48:1170-1173.

11. Silva RG. Disfagia neurogênica em adultos pós-acidente vascular encefálico: identificação e classificação. Dissertação de Mestrado, Universidade Federal de São Paulo, São Paulo 1997.

12. Gordon C, Hewer R L, Wade DT. Dysphagia in acute stroke. Br Med J 1987;295:411-414.

13. Mann G, Hankey GJ, Cameron D. Swallowing function after stroke. Stroke 1999;30: 744-748.

14. Veis SL, Logemann JA. Swallowing disorders in persons with cerebrovascular accident. Arch Phys Med Rehabil 1985;66:372-375. 